\title{
Pharmacy students' evaluation of an interprofessional prescribing workshop with final year medical and pharmacy students
}

\author{
Authors: Nicola Brown ${ }^{1}$, Kurt Wilson ${ }^{2}$, Ali Esmai ${ }^{3}$, Farhana Patel ${ }^{3}$. \\ 1.Manchester Pharmacy School \\ 2.Manchester Medical School \\ 3.Undergraduates at Manchester Pharmacy School, University of Manchester
}

Background: 54 fourth year pharmacy students enrolled on a patient safety optional module attended one prescribing day component with 5th year medical students. The compulsory prescribing day prepared medical students for a summative prescribing safety assessment to address prescribing safety and ability typical of foundation year 1 doctors. This assessment is a pass / fail requirement for the final year medical students. Pharmacy students were required to apply their medication safety knowledge to the interdisciplinary seminars and workshops and contribute to the case discussions. Interactive morning seminars on the BNF, fluids, controlled drugs and anticoagulation prepared students with knowledge to contribute to mixgroup case-based workshops on prescribing, prescription review, calculations, adverse drug reactions and drug monitoring. The workshops involved individual and group analysis of cases facilitated by tutors from a wide range of clinical specialties and disciplines.

Description of work: Two final year project students observed the first workshop, performed a literature review and created a questionnaire to evaluate the interprofessional prescribing day. The readiness for interprofessional learning questionnaire was adapted and combined with open questions to identify what students learnt from the experience, from other healthcare professionals and their perceived confidence in knowledge and interdisciplinary interaction. The questionnaire was retrospectively distributed. Data was entered into Excel for quantitative analysis. Open questions were thematically analysed to identify trends in responses.

Proposed evaluation: The response rate for pharmacy students was 38/54. The evaluation will evaluate the pharmacy students learning experience and allow the department to map the workshop outcomes onto the School interprofessional education strategy. The results will shape the future preparation ensuring intended prior knowledge and skills are tailored for students to optimally engage in the interprofessional learning experience. 